\title{
ARTICLE
}

\section{Reliability Analysis on the Container of Radioactive Material Transport Based on Monte Carlo}

\author{
Renze WANG ${ }^{1,2 *}$ \\ ${ }^{I}$ China Institute for Radiation Protection, Taiyuan, Shanxi Province, P. R. China \\ ${ }^{2}$ Department of Engineering Physics in Tsinghua University, Beijing, P. R. China
}

\begin{abstract}
The reliability of the container of radioactive material transport, container for short, is essential in radioactive material transport safety. Failure function of the container can be deduced by history records, experimental data or other means. Considering the randomness of main assessment parameters such as mechanical stress level and fire temperature, importance sampling MC (Monte Carlo) simulation method is used in this work to compile a computer program in MATLAB to calculate container failure probability in a certain CL (confidence level). The results show that MC method can represent objective uncertainty of assessment parameters and it is an effective complement to historical records and experiments.
\end{abstract}

\section{KEYWORDS: container of radioactive material transport, reliability, Monte Carlo, importance sampling}

\section{Introduction}

As an important aspect of nuclear safety, radioactive material transport safety has been widespread concerned internationally. Generally, radioactive material transport safety depends on the quality of the package. For safety, Regulations for the Safe Transport of Radioactive Material of IAEA takes the key of safe reliability for package design ${ }^{1)}$, so the reliability of the container is essential in transport safety.

Safety/risk assessments to transports of radioactive material in China are important tasks for Department of Health Physics and Nuclear Safety in China Institute for Radiation Protection. The risk of radioactive release in a transport of radioactive material is the product of container failure probability and the consequences of the accident. PSA (Probabilistic safety assessment) methodology was applied to assess risk of a transport of radioactive material in reference [2]. However, PSA is complicated to implement. If accuracy of the results is not strictly required, using the method proposed in this study, container failure probability can be directly estimated. Then according to the consequences of the accident, risk assessment of a transport can be evaluated.

Considering the randomness of main assessment parameters such as mechanical stress level and fire temperature, importance sampling MC simulation method is used in this work to compile a computer program in MATLAB to calculate container failure probability in a certain CL. This method is then compared with direct integral method.

\section{Container Failure Probability \\ 1. Failure Thresholds of the Container}

The so-called failure thresholds of the container are minimum temperature and minimum stress level in an accident which can lead to contents release in the container.

*Corresponding Author, E-mail:wrz198351@163.com
The values of failure thresholds are used to determine the container failure probability in an accident. Therefore, the values impact the final risk greatly.

There are three methods to achieve failure thresholds of the container. One is through experiments, another is through theoretical calculation, the third is through the synthesis of experiments and theoretical calculation.

Though values of failure thresholds of the container derived through experiments are credible, it costs too much. Generally, theoretical calculation or synthesis of experiments and theoretical calculation is adopted internationally. At present, the United States, Japan and some European countries have developed some corresponding softwares such as ANSYS, ANLOR and HEATING, the results of which agree with experimental data very well.

For examples, German CASTOR V/HAWC container used in HLLW (high level liquid waste) rail transport and French LR44 container used in HLLW road transport, their failure thresholds are shown in Table $\mathbf{1}^{3)}$.

Table 1 Failure thresholds of two HLLW containers

\begin{tabular}{|c|c|c|c|}
\hline & $\begin{array}{c}\text { Impact } \\
(\mathrm{km} / \mathrm{h})\end{array}$ & $\begin{array}{c}\text { Impenetration } \\
(\mathrm{km} / \mathrm{h})\end{array}$ & $\begin{array}{c}\text { Fire } \\
(\mathrm{min})\end{array}$ \\
\hline CASTOR & 88.7 & $>79.0$ & $>180$ \\
\hline LR44 & 90.4 & $>50.0$ & $>120$ \\
\hline
\end{tabular}

\section{Stress-Strength Model}

Stress-strength model (as shown in Fig. 1) has been world-widely studied in reliability engineering. The model was first proposed by Professor Birnbaum in University of California-Berkeley in $1955^{4)}$, and it has been widely used in many fields especially in structural analysis and aviation industry.

Generally, physical quantities impacting on systems or components such as stress, pressure, temperature are called stress of systems or components. The extent of systems or components to withstand some stress is called the strength of systems or components. If the strength is greater than the 


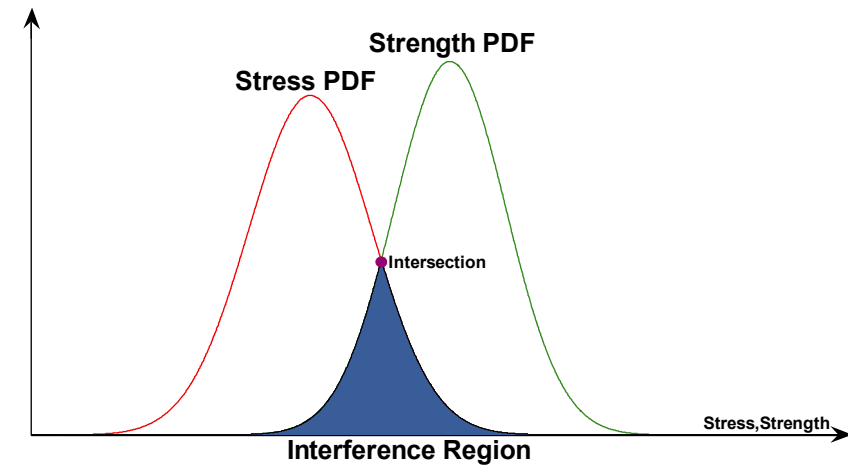

Fig. 1 Strength mode

stress, i.e. the stress is in the load range, integrity of the system or component can be maintained, the system or component is able to complete the required functions and which is known as success. If the strength is less than the stress, the stress will destroy functions or structures of the system or component, and then required functions can not be completed, which is known as failure. Stress and strength are random variables, which are subjected to statistical distributions and both of them are regarded to be subjected to normal distribution in many cases.

Calculation of container failure probability can be based on the stress-strength model.

\section{Probabilistic Distribution of Stress and Strength}

Taking rail/road transports as examples, according to possible impacting modes, there are five conditions involved in rail/road accidents, i.e. fire, impact, pressure, impenetration and submersion. Failure analysis of CASTOR and LR44 containers show that whether road transport or rail transport only impact and fire can lead to radioactive release accidents ${ }^{3}$. Characteristic parameters of impact intensity are impact speed and angle against the surface. Characteristic parameters of fire intensity are fire temperature, burning time and the distance to the fire. For the reliability calculation of the container, only the most important or synthetic effective characteristic parameter of an intensity is considered while the other parameters are default. For examples, for impact only mechanical stress against the container surface is considered while for fire only fire temperature is considered, burning time is default long enough and the distance to the fire is default close enough. Mechanical stress and fire temperature are both called stress as mentioned above.

Stress distribution generally accords with normal distribution and stress is denoted by random variable $X$ where $X$ is supposed to be subjected to a normal distribution whose mean is $\mu$ and whose standard deviation is $\sigma$ and the probability density of $x$ is $n(x)$. Actually, both of mechanical stress and fire temperature to the container are non-negative. In most normal circumstances, there is no mechanical stress or fire to the container, i.e. the probability density of 0 is the maximum, so stress is subjected to a normal distribution which has only positive interval. That is to say, actually, above-mentioned $\mu=0$ for $x \geq 0$ and for number normalization, the probability density of $x$ is $f(x)=2 n(x)$. The relation between $f(x)$ and $n(x)$ is shown in Fig. 2. To facilitate the calculation, "standard deviation" of $f(x)$ is not standard deviation in real meaning, and it is expressed as the standard deviation $\sigma$ of $n(x)$.

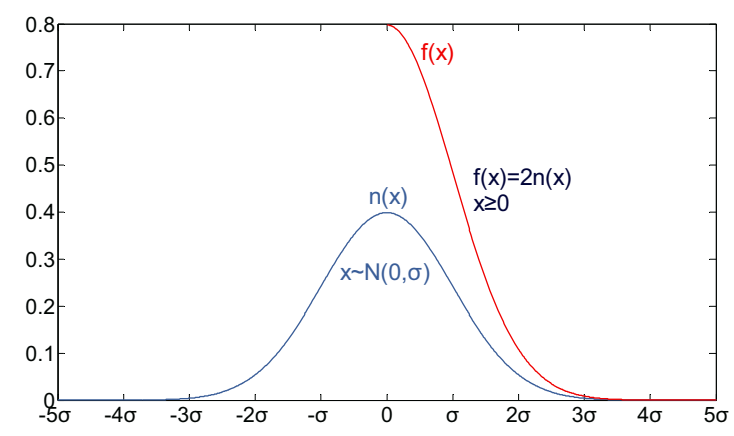

Fig. 2 Relation between $f(x)$ and $n(x)$

Container failure threshold is equivalent to the strength in the stress-strength model, which is denoted by $Y$, and $Y$ is subjected to a normal distribution in general.

PDF (probability density function) of the thermal stress against FCo70-YQ model package and its failure threshold are shown in Fig. 3. (Unit of horizontal abscissa is ${ }^{\circ} \mathrm{C}$ in the figure, and vertical ordinate is corresponding to probability density.)
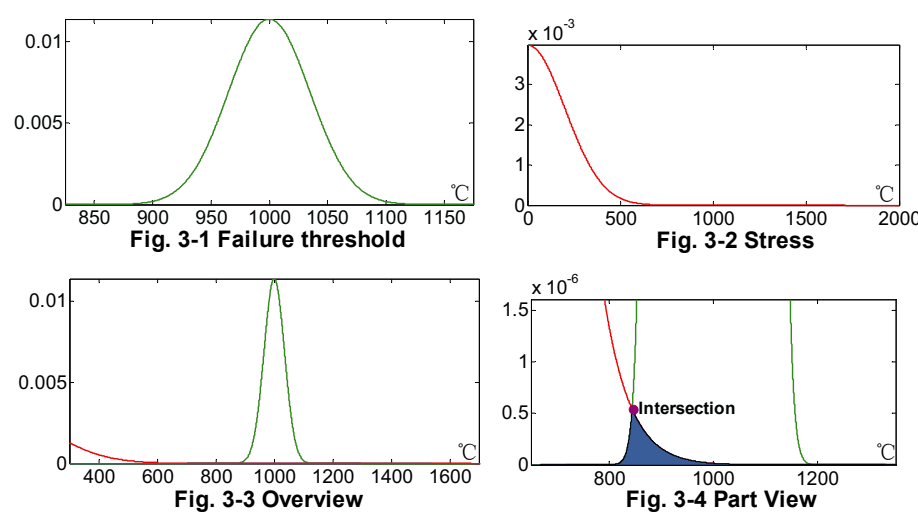

Fig. 3 PDF of the stress and failure threshold

FCo70-YQ model package which is manufactured and named by a Chinese company belongs to $\mathrm{B}(\mathrm{U})$ model package, and it is designed as a equipment in special use for transport of ${ }^{60} \mathrm{Co}$ radioactive source which is used in ${ }^{60} \mathrm{Co}$ teletherapy machine and blood irradiation instrument. The package applies to road, rail and ship transports, package quantity in a transport is not limited, and the designed service life of the package is 20 years.

Failure threshold is achieved by combined methods of thermal experiment and simulation calculation by ANSYS11.0 software ${ }^{5)}$. Experimental conditions and acceptance criteria are according to Regulations for the Safe Transport of Radioactive Material (GB11806-2004, a Chinese national standard) ${ }^{6}$, and Regulations for the Safe Transport of Radioactive Material (TS-R-1) of IAEA ${ }^{1)}$ is 
consulted.

Fig. 3-1 shows PDF curve of the failure threshold, Fig. 3-2 shows PDF curve of the stress and Fig. 3-3 shows an overview of two curves plotted in the same reference frame. Probability density of the stress near the mean of the failure threshold is so small that the stress curve is close to horizontal axis. Fig. 3-4 shows part view of interference region whose area in black shadow is the container failure probability for this stress.

\section{Expression of Container Failure Probability}

In the basic theory of structure system reliability, failure function represents system failure surface. Failure surface i.e. limit state surface divides the state space into two parts of failure space $\omega_{f}$ and safe space $\omega_{s}$. The stress against the container is denoted by $X$ while the threshold is denoted by $Y$, both of which are vectors, failure function $g(X)$ is shown as follows:

$$
\begin{gathered}
g(X)=Y-X \\
g(X)>0, X \in \omega_{s} \\
g(X)<0, X \in \omega_{f}
\end{gathered}
$$

The reliability of the container can be expressed as follows:

$$
R_{s}=1-P_{f}=1-\int_{\omega_{f}} f(X) \mathrm{d} X
$$

where

$f(X)$ is PDF of the stress, impact stress is denoted by $x_{1}$ and fire temperature is denoted by $x_{2}$.They are random variables independent of each other. $f(X)$ is shown as follows:

$$
f(X)=f\left(x_{1}\right) f\left(x_{2}\right)
$$

The evaluation of container failure probability $P_{f}$ is the key point:

$$
P_{f}=\int_{\omega_{f}} f(X) \mathrm{d} X=\int_{g(X)<0} f(X) \mathrm{d} X
$$

\section{Importance Sampling Monte Carlo Method}

The integral equation (5) is multidimensional, MC method is primarily used in this study to evaluate the equation, which is equivalent to equation (6).

$$
P_{f}=\int_{\Omega} I[g(X)] f(X) \mathrm{d} X
$$

In equation (6), $I[g(X)]$ is an indicative function. If $g(X)<0$, container failure is indicated, then $I[g(X)]=1$;If $g(X)>0$, container reliability is indicated, then $I[g(X)]=0$. Integral domain $\Omega$ denotes the entire space. As container failure probability is usually very small, in a direct sampling method to calculate equation (6) large numbers of sampling need be carried out to achieve the specified error level. After each sampling container failure is to be determined, the computation work is too much. The best way to solve this problem is to replace direct sampling method with importance sampling method. In an importance sampling method the statistical quantity $I[g(X)] \frac{f(X)}{h(X)}$ replaces $I[g(X)]$, so equation (6) can be expressed as follows:

$$
P_{f}=\int_{\Omega} I[g(X)] \frac{f(X)}{h(X)} h(X) \mathrm{d} X
$$

Here $h(X)$ is an importance sampling function and it is nonnegative and is subjected to number normalization. As an unbiased estimate of the container failure probability the mean of $I[g(X)] \frac{f(X)}{h(X)}$ is shown as follows:

$$
\widehat{P}_{f}=\frac{1}{\mathrm{~N}} \sum_{i=1}^{N} I\left[g\left(\mathrm{x}_{i}\right)\right] \frac{f\left(\mathrm{x}_{i}\right)}{h\left(\mathrm{x}_{i}\right)}
$$

The key point of importance sampling MC method is to choose a suitable importance sampling density function to effectively reduce the variance. The most commonly used method is to adopt a normal distribution PDF as an importance sampling density function. Its mean is the designed point and its variance is not less than the variance of the original random variable $X$. In this sampling method, large numbers of sampling points are located near the designed point. If non-linear degree of the failure surface is not very high, sampling efficiency is usually about $50 \%$.

CUL (confidence upper limit) at a certain CL can be calculated as the following steps ${ }^{7}$ :

(1) $M$ container failure probabilities

$\widehat{P}_{f}^{j}, j=1,2, \cdots, M$ are calculated by importance sampling MC method.

(2) $\widehat{P}_{f}^{j}, j=1,2, \cdots, M$ are sorted by ascending order as follows:

$$
\widehat{P}_{f}^{(1)} \leq \widehat{P}_{f}^{(2)} \leq \cdots \leq \widehat{P}_{f}^{(k)} \leq \cdots \leq \widehat{P}_{f}^{(M)}
$$

$P\left(P_{f} \leq \hat{P}_{f}^{(k)}\right) \approx \frac{k}{M}$, so $\hat{P}_{f}^{(k)}$ is the approximate CUL of $P_{f}$ at the CL of $\frac{k}{M}$.

\section{Compiling of MATLAB Program and Calculation of Container Failure Probability \\ 1. Compiling of MATLAB Program}

Based on the above basic principles, a MATLAB program is compiled to calculate container failure probability. The importance sampling density function is a normal distribution PDF, its mean is the intersection (black dot, as shown in Fig. 1 or Fig. 3) near the origin between failure threshold curve and stress curve while its variance is equal to the variance of the stress. All normal distributions are standardized to standard normal distribution in the program before calculation.

The GUI (graphical user interface) of compiled MATLAB program is shown in Fig. 4.

In the upper part of the GUI is a parameter input area. In the middle part of the GUI is an operation area. In the lower part of the GUI is a calculation area. The specified function of each part is shown in Table 2. (In order to write simply and conveniently, name of each PB (push button) or ET (edit 


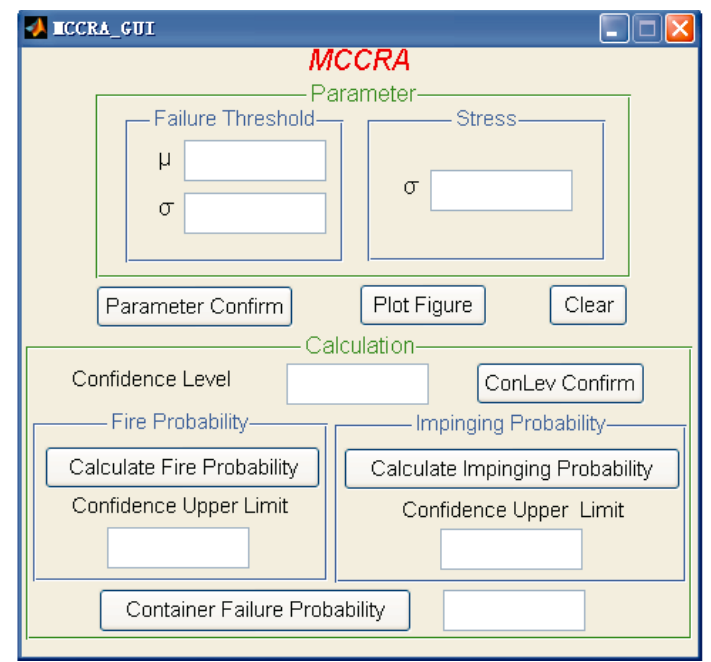

Fig. 4 GUI of container failure probability calculation program

text) is abbreviated as the first letter set of the name's words. For example, CIP is the abbreviation of "Calculate Impinging Probability").

Table 2 Function list of each part of the GUI

\begin{tabular}{|c|c|c|}
\hline \multicolumn{2}{|c|}{ PB/ET } & Function \\
\hline \multirow{2}{*}{ FT } & $\mu$ & Input the mean of the failure threshold. \\
\cline { 2 - 4 } & $\sigma$ & $\begin{array}{r}\text { Input the standard deviation of the } \\
\text { failure threshold. }\end{array}$ \\
\hline S & $\sigma$ & Input the standard deviation of the stress. \\
\hline \multicolumn{2}{|c|}{ PC } & Confirm the input parameters just above. \\
\hline \multicolumn{2}{|c|}{ PF } & Plot figures. \\
\hline \multicolumn{2}{|c|}{ C } & Clear data in the workspace. \\
\hline \multicolumn{2}{|c|}{ CL } & Input a CL. \\
\hline \multicolumn{2}{|c|}{ CC } & Confirm the CL. \\
\hline \multirow{2}{*}{ FP } & CFP & Show fire CUL. \\
\cline { 2 - 4 } CUL & Calculate impinging CUL. \\
\hline \multirow{2}{*}{ IP } & CIP & Show impinging CUL. \\
\cline { 2 - 3 } & CUL & Calculate the total failure probability. \\
\hline \multicolumn{2}{|c|}{ CFP } &
\end{tabular}

According to experiments and simulation calculations by software, fire temperature, impact stress and corresponding failure thresholds of FCo70-YQ model package are presented in Table 3, calculation results are also in the table.

\section{Comparison between Two Methods}

Direct integral method for integration of Eq. (5) is also used in MATLAB and then container failure probability can be achieved. Adopting basic data in Table 3, the comparison of the results obtained by the MC method and direct integral method is shown in Table 4, which presents that relative error of two methods is less than $1 \%$.
Table 3 Calculation example

\begin{tabular}{|c|c|c|c|c|}
\hline \multirow{2}{*}{} & \multicolumn{2}{|c|}{ FT } & \multicolumn{2}{c|}{ CL } \\
\cline { 2 - 5 } & \multirow{2}{*}{$\mathrm{m}$} & \multirow{2}{*}{$\mathrm{s}$} & $80 \%$ & $90 \%$ \\
\cline { 4 - 5 } & & & \multicolumn{2}{c|}{ CUL $(\mathrm{e}-5)$} \\
\hline Fire $\left({ }^{\circ} \mathrm{C}\right)$ & 1000 & 35 & 2.964 & 2.966 \\
\hline Impact $(\mathrm{kPa})$ & 3600 & 70 & 4.730 & 4.735 \\
\hline
\end{tabular}

Table 4 Comparison between two methods

\begin{tabular}{|c|c|c|c|c|c|}
\hline & \multicolumn{2}{|c|}{$\mathrm{CL}$} & \multirow{3}{*}{$\begin{array}{c}\text { Direct } \\
\text { Integral } \\
\text { Method } \\
\text { (e-5) }\end{array}$} & \multicolumn{2}{|c|}{$\mathrm{CL}$} \\
\hline & $80 \%$ & $90 \%$ & & $80 \%$ & $90 \%$ \\
\hline & \multicolumn{2}{|c|}{ CUL(e-5) } & & \multicolumn{2}{|c|}{ Relative Error } \\
\hline Fire & 2.964 & 2.966 & 2.950 & $0.47 \%$ & $0.54 \%$ \\
\hline Impact & 4.730 & 4.735 & 4.699 & $0.66 \%$ & $0.77 \%$ \\
\hline
\end{tabular}

\section{Conclusion}

The obtained outcome of the importance sampling MC method agrees with the outcome of an integral method, therefore the study shows that the container reliability is very high. The CUL of the container failure probability at a certain CL can be also achieved by MC method. Though computation time increases, stochastic characteristics of the assessment parameters are represented very well, so it is an effective complement to historical records and experiments.

\section{References}

1) Regulations for the Safe Transport of Radioactive Material (2005 Edition),IAEA Safety Standards Series No. TS-R-1, International Atomic Energy Agency (IAEA), (2005).

2) Bing Zhao, Jiangang Zhang, Xuexin Wang, et al., "PSA method in transport of radioactive material," The Fourth Symposium of Forum for Radiation Protection at Beginning of the 21st Century, Beijing, China, June 9-10,2005,274(2005), [in Chinese].

3) A.W.Dennis, J.T.Foley, W.F.Hartman et al., Severities of transportation accidents involving large packages, SAND-77-0001, U.S. Sandia Laboratories, (1978).

4) Z.M.Birnbaum. "On a use of the Mann-Whitney Statistic," Proceedings of the Third Berkeley Symposium on Mathematical Statistics and Probability, Berkeley, California, December, 1954, July and August, 1955, Volume I, 13 17(1956).

5) Kent Lawrence, ANSYS Workbench Tutorial Release 11, Schroff Development Corporation, Mission, Kansas, 2007

6) Regulations for the Safe Transport of Radioactive Material, GB11806-2004, General Administration of Quality Supervision, Inspection and Quarantine of P.R.China, Standardization Administration of P.R.China, (2004).

7) Gang Xiao, Tiantuo Li, Monte Carlo Method in System Reliability Analyzing, Science Press, Beijing, 140(2003), [in Chinese] 